\title{
Bloqueo del plano del erector espinal como analgesia en fracturas costales múltiples unilaterales
}

\author{
J. F. Vargas Silva', C. E. Vela Izquierdo², L. N. Ricaurte Gracia ${ }^{3}$, J. O. Castillo Rodríguez y A. I. Aparicio Negrete ${ }^{5}$
}

${ }^{1}$ Anestesiólogo. Especialista en Medicina del Dolor y Cuidados Paliativos. Universidad El Bosque. Hospital

Pablo Tobón Uribe. Colombia. ${ }^{2}$ Médico Residente de Anestesia. Analgesia y Reanimación del Instituto

Nacional Materno Perinatal. Perú. ${ }^{3}$ Anestesióloga. Fellowship Medicina del Dolor y Cuidados Paliativos.

Universidad El Bosque. Colombia. ${ }^{4}$ Anestesiólogo. Fellowship Medicina Paliativa y del Dolor. Hospital

General de Occidente. México. ${ }^{5}$ Médico Residente de Anestesiología. Universidad de Caldas. Colombia

\section{ABSTRACT}

Rib fractures are common lesions, being present in $10 \%$ of patients with closed chest trauma. It is not a serious pathology, but it is an indicator of morbidity and mortality. The risk complications, especially the pulmonary mechanics compromise of the affected patient makes it imperative to achieve optimal pain control. What makes multimodal analgesia an important pillar in the management of this entity, within this, the blocks with local anesthetic use, play an important role.

Recently, the erector spinae plan block (ESP) with different analgesic purposes has been described given its anatomical coverage. This technique has been implemented for management of several painful syndromes and compromises with considerable success.

This report attempts to describe the erector spinae plan block for analgesic control in a patient with several thorax fractures secondary to multiple trauma.

Key words: Regional Anesthesia, analgesia, rib fracture, chest pain, thoracic surgery.

\section{INTRODUCCIÓN}

Las fracturas costales son lesiones comunes después de una contusión torácica. Se presenta en, aproximadamente, el $10 \%$ de los pacientes tras un trauma cerrado de tórax (1); rara vez son mortales, pero pueden ser un marcador de una lesión visceral grave

Vargas Silva JF, Vela Izquierdo CE, Ricaurte Gracia LN, Castillo Rodriguez JO y Aparicio Negrete Al. Bloqueo del plano del erector espinal como analgesia en fracturas costales múltiples unilaterales. Rev Soc Esp Dolor 2019;26(3):199-202.

\section{RESUMEN}

Las fracturas costales son lesiones comunes, estando presentes en el $10 \%$ de los pacientes con trauma cerrado de tórax. No es una patología grave, pero puede comportarse como un buen indicador de morbimortalidad en el paciente que las padece. El riesgo de complicaciones, especialmente el compromiso de la mecánica pulmonar del paciente afectado, hace imperativo lograr un control óptimo del dolor, lo que convierte a la analgesia multimodal en un pilar importante en el manejo de esta entidad, constituyendo el uso de bloqueos con anestésico local un aspecto importante.

Recientemente se ha descrito la utilización del bloqueo del músculo erector espinal con fines analgésicos dado su alcance anatómico; esta técnica se ha implementado para el manejo de varios síndromes dolorosos con buenos resultados.

Este artículo describe el uso del bloqueo del erector espinal para el control analgésico en un paciente con fracturas de tórax secundarias a un politraumatismo.

Palabras clave: Anestesia regional, analgesia, fractura costal, dolor torácico, cirugía torácica. dentro del abdomen o tórax en traumas graves, como la fractura de la primera costilla (2-4). Según el estudio de Sammy y cols., basado en los datos obtenidos del UK Trauma Audit, las fracturas de la primera costilla se comportan como un predictor significativo de gravedad de la lesión y el politrauma (puntuación de gravedad de la lesión o ISS > 15 por sus siglas en inglés] (3). 
Dentro de las causas más frecuentes de este tipo de lesiones son los accidentes automovilísticos en los adultos, las actividades recreativas o deportivas y los traumas no accidentales en los jóvenes. Asimismo, las fracturas costales podrían ser secundarias a un compromiso oncológico [5].

El diagnóstico suele hacerse con radiografías de tórax anteroposteriores (AP) y laterales, sin embargo la tomografía computarizada de tórax es el examen radiológico más sensible para detectarlas $(6,7)$.

Dado el cuadro clínico, la repercusión física y el riesgo de complicaciones, especialmente la restricción funcional por compromiso de la mecánica ventilatoria, hace necesario un control óptimo del dolor $[8,9]$. La analgesia multimodal, no solo con medicamentos sino con procedimientos que ayuden a disminuir el consumo de fármacos y logren modular el dolor, son primordiales. Dentro de ellos, los bloqueos nerviosos intercostales, el bloqueo epidural torácico y el bloqueo paravertebral pueden proporcionar alivio del dolor pero no son inocuos (10), dado el riesgo de inyección intravascular, neumotórax y, en caso de fracturas costales múltiples, la necesidad de realizar el procedimiento en varios niveles, incluso llegando a requerir dosis considerables del anestésico local (11-14).

Anatómicamente, las costillas y los tejidos adyacentes se inervan por los nervios torácicos, que se ramifican en ramos ventrales y dorsales después de pasar el foramen intervertebral. Los ramos ventrales se convierten en los nervios intercostales, que recorren la profundidad de la membrana intercostal interna y el plano entre el músculo intercostal interno y el íntimo, inervando así la mayor parte de la pared torácica lateral y anterior (1517). La rama dorsal, por su lado, viaja posteriormente y asciende al músculo erector espinal y se divide en ramas lateral y medial; esta última, asciende a través de los músculos romboides mayor y trapecio e inerva la pared torácica posterior. El bloqueo del plano del erector espinal, o ESP por sus siglas en inglés, es una técnica reciente (15), simple y segura, en la analgesia torácica, tanto en el dolor neuropático crónico como en el dolor posquirúrgico o postraumático agudo (fracturas costales simples o múltiples) $(16,18-20)$. La inyección en esta región muestra diseminación del medicamento administrado en, al menos, cuatro niveles vertebrales por encima y tres niveles vertebrales por debajo del punto de inyección, en dirección cefalocaudal (15), paravertebral, e incluso hasta el origen de los nervios intercostales y ramos dorsales, lo que resulta en una buena analgesia del hemitórax en su pared lateral, anterior y posterior, lo que ofrece una explicación lógica para los amplios cambios sensoriales y analgésicos encontrados posterior al procedimiento (18)(21).

\section{DESCRIPCIÓN DEL CASO}

Paciente de 28 años, remitido al hospital por accidente de tránsito en calidad de conductor. Colisiona a alta velocidad, sufriendo politrauma grave. La radiografía de tórax de ingreso muestra fractura de los arcos costales posteriores del lado izquierdo desde la segunda a la séptima costilla a menos de $2,5 \mathrm{~cm}$ de la apófisis transversa y de la quinta a la séptima a $3 \mathrm{~cm}$ de la apófisis transversa. Además, se detecta fractura del arco costal anterior de la primera, segunda y tercera costilla, y en el arco costal lateral de la sexta costilla.

El paciente requirió manejo en la unidad de cuidados intensivos por diagnóstico de trauma encefalocraneano grave y deterioro neurológico secundario a contusiones hemorrágicas intraparenquimatosas y gangliobasales izquierdas, con daño axonal difuso. Tuvo también contusión pulmonar y neumonía por aspiración asociado a insuficiencia respiratoria hipoxémica, por lo cual requirió ventilación mecánica y soporte hemodinámico.

La evolución clínica fue satisfactoria y se solicitó interconsulta a Clínica del Dolor para optimizar manejo analgésico, con el fin de facilitar la extubación, considerando el compromiso de la mecánica ventilatoria secundaria a la patología de ingreso. En el momento de la valoración, se encuentra al paciente con un consumo de 12,5 mg de metadona vía oral cada 6 horas, sin buen control del dolor y sin adecuado acople al ventilador, por lo que se decide realizar una técnica analgésica regional. Teniendo en cuenta las fracturas costales, se sugirió inicialmente un bloqueo de los nervios intercostales, sin embargo la región dolorosa era muy amplia y el riesgo de neumotórax considerable. Igualmente, la inyección torácica epidural o paravertebral se descartó al considerarse demasiado invasiva y poco viable dado su estado. Finalmente, se optó por un catéter ESP guiado por ultrasonido, según la técnica descrita por Forero y cols. (15). El procedimiento se realizó cuatro días después del ingreso en el hospital. Se colocó al paciente en posición de decúbito lateral, usando el transductor lineal de alta frecuencia (5-15 Mhz), se visualizó inicialmente en eje largo la apófisis espinosa de T5, posteriormente se giró el transductor al eje corto, se identificaron tres músculos superficiales (trapecio, romboides mayor y erector espinal] y con una aguja tuohy calibre $18 \mathrm{G}$ de $8 \mathrm{~cm}$ de largo se realizó la punción en plano con el transductor, en dirección cefálico-caudal, buscando el plano interfascial superficial a las apófisis transversa de T5. Con el ultrasonido se localizó la punta de la aguja en el plano interfascial entre los músculos romboides mayor y erector de la columna, inyectando un total de $20 \mathrm{ml}$ de bupivacaína al 0,5 \% (Figura 1) y se avanzó el catéter en el mismo lugar y bajo visión ecográfica. Se fijó el catéter con Tegaderm ${ }^{\circledR}$ transparente (Figura 2), pautando infusión a $4 \mathrm{ml} /$ hora de bupivacaína sin epinefrina al 0,1\%.

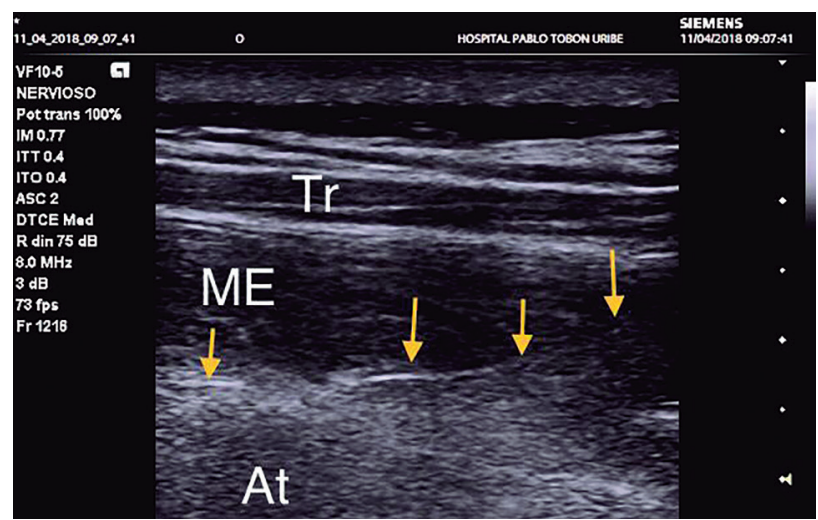

Catéter (Flechas Amarillas). At: apófisis transversa de T5. Tr: músculo trapecio. ME: músculo erector de la espina.

Fig. 1. Imagen ecográfica del catéter en el plano del erector. 


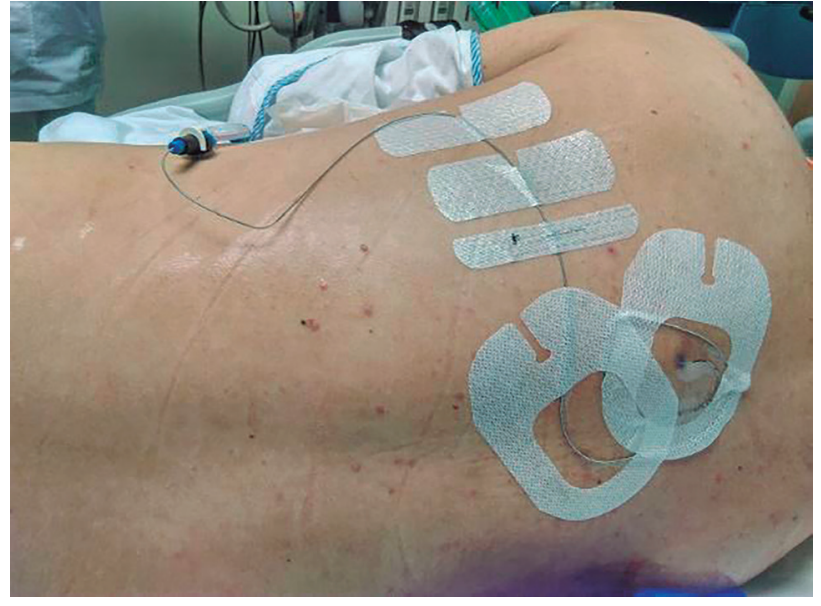

Fig. 2. Catéter ESP fijado.

Se hizo seguimiento durante cinco días, evidenciando mejoría en parámetros ventilatorios y acople al ventilador, además de la disminución del soporte hemodinámico; del primer al cuarto día el paciente permaneció intubado, con una escala CPOT (Critical-Care Pain Observation Tool] de 2 puntos (0-8 puntos] (22). El quinto día se realizó la extubación programada; 12 horas después se retiró la infusión analgésica y 24 horas postextubación se interrogó al paciente para valorar intensidad del dolor. En ese momento, y sin necesidad adicional de medicamentos opiáceos, el paciente manifestó dolor moderado en la región de las fracturas costales, calificándolo en 4 puntos usando la escala numérica análoga de 0 a 10 . Adicionalmente, se hizo una evaluación sensitiva del cubrimiento analgésico, dibujando la zona con sensación de analgesia residual referida por el mismo paciente (Figura 3).

Posterior a esta última valoración, se inició tratamiento con paracetamol $325 \mathrm{mg}+$ hidrocodona $5 \mathrm{mg}$ cada 8 horas, obteniendo adecuado control del dolor.

\section{DISCUSIÓN}

El control óptimo del dolor por fracturas costales secundarias a trauma representa un reto importante, especialmente si la mecánica ventilatoria está comprometida y se intenta disminuir el consumo de analgésicos opioides. Se crea entonces una disyuntiva en la que se analizan los beneficios de la analgesia farmacológica versus las posibles técnicas de analgesia regional ofertadas por el médico especialista de dolor; aplicando una verdadera terapia de analgesia multimodal logrando reducir al máximo las dosis de medicamentos, principalmente opioides, y consecuentemente disminución en los efectos secundarios asociados a posologías elevadas de los mismos. Teniendo en cuenta esto, el empleo de una técnica de analgesia regional es una opción a considerar.

Entre estas existen diversas opciones, como el bloqueo del músculo erector espinal, que es una técnica relativamente nueva (15). En esta técnica, la disemi-

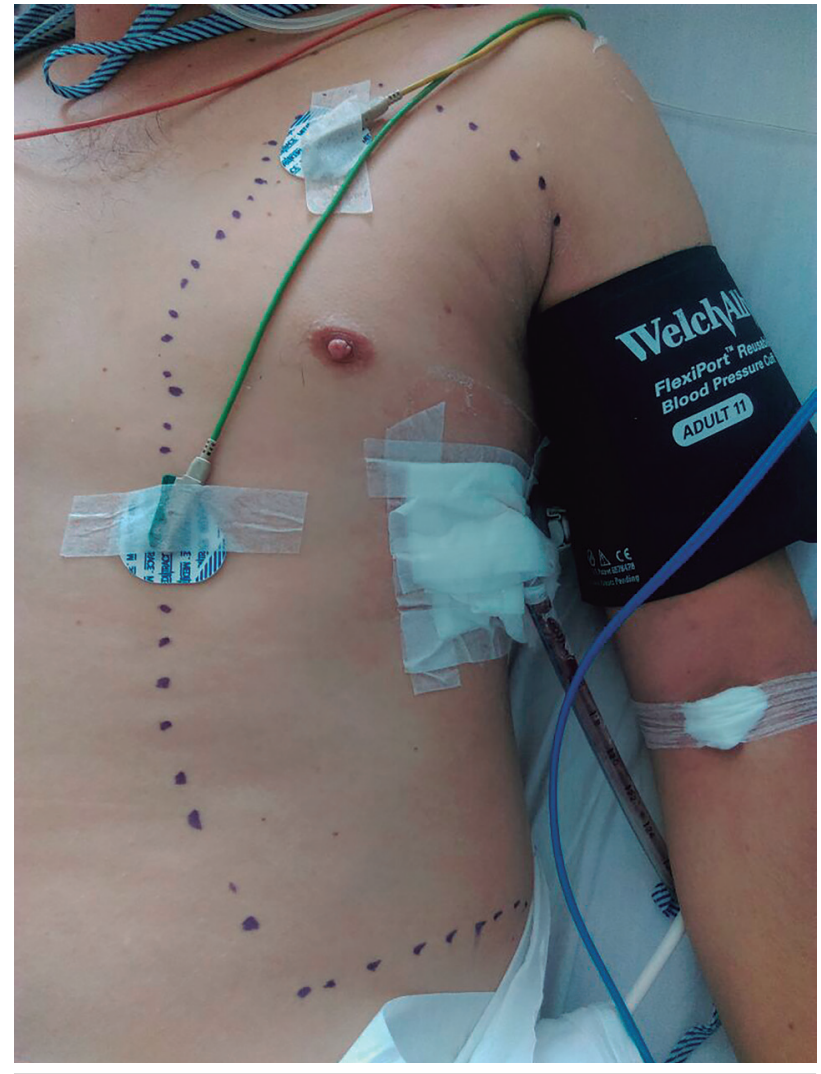

Fig. 3. Evaluación sensitiva del cubrimiento analgésico.

nación del anestésico local a niveles tanto superiores como inferiores del sitio de punción, así como en dirección al foramen vertebral, sugieren un beneficio en el manejo analgésico de dolores torácicos $(15,21)$. Dada la alta frecuencia de fracturas costales y el efecto encontrado al usar esta técnica, se sugiere considerar este abordaje en pacientes con traumatismo torácico. Además, el margen de seguridad del procedimiento es alto y principalmente, la baja complejidad hace que realizarlo sea relativamente simple, incluso en pacientes intubados $[23,24)$.

El empleo de ultrasonidos ofrece seguridad clínica al paciente y corrobora la efectividad de la técnica seleccionada.

\section{CONCLUSIONES}

El uso de una técnica de analgesia regional enriquece el manejo médico del paciente politraumatizado, disminuye el consumo de medicamentos y ofrece un control óptimo analgésico.

El bloqueo ESP es una alternativa que, a pesar de ser descrito recientemente, se ha usado ampliamente en varios tipos de lesiones y procedimientos quirúrgicos, mostrando ser una técnica segura y que aporta un beneficio clínico importante, lo que hace pensar que no solo en traumas, sino que también en lesiones más complejas como dolores neuropáticos, cirugías abdo- 
minales o torácicas o incluso en entidades como el síndrome doloroso regional complejo, brindarían una analgesia significativa.

\section{FINANCIACIÓN}

Los autores no recibieron patrocinio para llevar a cabo este artículo.

\section{CONFLICTO DE INTERESES}

Los autores declaran no tener ningún conflicto de intereses.

\section{BIBLIOGRAFÍA}

1. Kasotakis G, Hasenboehler EA, Streib EW, Patel N, Patel $\mathrm{MB}$, Alarcon L, et al. Operative fixation of rib fractures after blunt trauma. J Trauma Acute Care Surg 2017;82(3):61826. DOI: 10.1097/TA.0000000000001350.

2. Murphy CE, Raja AS, Baumann BM, Medak AJ, Langdorf MI, Nishijima DK, et al. Rib Fracture Diagnosis in the Panscan Era. Ann Emerg Med 2017;70(6):904-9. DOI: 10.1016/j. annemergmed.2017.04.011.

3. Sammy IA, Chatha H, Lecky F, Bouamra O, Fragoso-lñiguez M, Sattout A, et al. Are first rib fractures a marker for other lifethreatening injuries in patients with major trauma? A cohort study of patients on the UK Trauma Audit and Research Network database. Emerg Med J 2017;34(4):205-11. DOI: 10.1136/emermed-2016-206077.

4. Yonezawa N, Nakayama Y, Takei T, Toh M, Asano M, Imamura $T$, et al. Fatal delayed rupture of the subclavian artery in a patient with first-rib fracture caused by blunt trauma. Clin Case Reports 2017;5(3):260-3. DOI: 10.1002/ccr3.823.

5. Ochi K, Furuya T, Ikari K, Taniguchi A, Yamanaka H, Momohara S. Sites, frequencies, and causes of self-reported fractures in 9,720 rheumatoid arthritis patients: a large prospective observational cohort study in Japan. Arch Osteoporos 2013;8(1-2):130. DOI: 10.1007/s11657-013-0130-7.

6. Magu S, Yadav A AS. Computed tomography in blunt chest trauma. Indian J Chest Dis Allied Sci 2009;51(2):75-81.

7. Henry TS, Kirsch J, Kanne JP, Chung JH, Donnelly EF, Ginsburg ME, et al. ACR Appropriateness Criteria $₫$ Rib Fractures. J Thorac Imaging 2014;29(6):364-6. DOI: 10.1097/ RTI.0000000000000113.

8. Cataneo AJM, Cataneo DC, de Oliveira FH, Arruda KA, El Dib R, de Oliveira Carvalho PE. Surgical versus nonsurgical interventions for flail chest. Cochrane Database Syst Rev 2015; 29;(7): CD009919. DOI: 10.1002/14651858.CD009919.pub2.

9. Majercik S, Pieracci FM. Chest Wall Trauma. Thorac Surg Clin 2017;27(2):113-21. DOI: 10.1016/j.thorsurg.2017.01.004.
10. Ho AM-H, Karmakar MK, Critchley LAH. Acute pain management of patients with multiple fractured ribs. Curr Opin Crit Care 2011;17(4):323-7. DOI: 10.1097/MCC.0b013e32 8348bf6f.0075198-201108000-00003

11. Hwang EG, Lee Y. Effectiveness of intercostal nerve block for management of pain in rib fracture patients. J Exerc Rehabil 2014;10(4):241-4. DOI: 10.12965/jer.140137.

12. Mohta M, Verma P, Saxena AK, Sethi AK, Tyagi A, Girotra G. Prospective, Randomized Comparison of Continuous Thoracic Epidural and Thoracic Paravertebral Infusion in Patients With Unilateral Multiple Fractured Ribs-A Pilot Study. J Trauma Inj Infect Crit Care 2009;66(4):1096-101. DOI: 10.1097/ TA.Ob013e318166d76d.

13. Karmakar MK, Ho AM-H. Acute Pain Management of Patients with Multiple Fractured Ribs. J Trauma Inj Infect Crit Care 2003;54(3):615-25. DOI: 10.1097/01. TA.0000053197.40145.62.

14. Malekpour M, Hashmi A, Dove J, Torres D, Wild J. Analgesic Choice in Management of Rib Fractures. Anesth Analg 2017; 124(6): 1906-11. DOI: 10.1213/ANE.0000000000002113.

15. Forero M, Adhikary SD, Lopez H, Tsui C, Chin KJ. The Erector Spinae Plane Block. Reg Anesth Pain Med 2016;41(5):6217. DOI: $10.1097 / A A P .0000000000000451$.

16. Hamilton DL, Manickam B. Erector spinae plane block for pain relief in rib fractures. Br J Anaesth 2017;118(3):4745. DOl: 10.1093/bja/aex013.

17. Waxenbaum JA, Futterman B. Anatomy, back, thoracic vertebrae. StatPearls 2018. Available from: http://www.ncbi. nlm.nih.gov/pubmed/29083651

18. Forero M, Rajarathinam M, Adhikary S, Chin KJ. Continuous Erector Spinae Plane Block for Rescue Analgesia in Thoracotomy After Epidural Failure. A A Case Reports 2017;8(10):2546. DOI: 10.1213/XAA.0000000000000478.

19. Kumar A, Hulsey A, Martinez-wilson H, Kim J, Gadsden J. The Use of Liposomal Bupivacaine in Erector Spinae Plane Block to Minimize Opioid Consumption for Breast Surgery: A Case Report. A A Pract 2018;10(9):239-41. DOI: 10.1213/XAA.0000000000000674.

20. Veiga M, Costa D, Brazão I. Bloqueo en el plano del músculo erector de la columna para mastectomía radical: ¿una nueva indicación? Rev Esp Anestesiol Reanim 2018;65(2):112-5. DOI: 10.1016/j.redar.2017.08.004.

21. Josh Luftig P, Mantuani D, Herring AA, Dixon B, Clattenburg E, Nagdev A. The authors reply to the optimal dose and volume of local anesthetic for erector spinae plane blockade for posterior rib fractures. Am J Emerg Med [Internet] 2018;36(6):1103-4. DOI: 10.1016/j. ajem.2018.03.051.

22. El-Boghdadly K, Pawa A. The erector spinae plane block: plane and simple. Anaesthesia 2017;72(4):434-8. DOI: 10.1111/anae.13830.

23. Forero M, Rajarathinam M, Adhikary S, Chin KJ. Erector spinae plane (ESP) block in the management of post thoracotomy pain syndrome: A case series. Scand $J$ Pain [Internet]. 2017;17:325-329. doi: 10.1016/j. sjpain.2017.08.013. 